\title{
The Effect of Action Observation Training with Acoustic Stimulation on Balance and Gait in Stroke Patients
}

\author{
Young-Mi Kim, PT, $\mathrm{PhD}^{\dagger} \cdot$ Ho-Jeong Lee, PT, MS ${ }^{1}$ - Jong-Su Lee, PT, MS ${ }^{1}$ \\ Department of Physical Therapy, Uiduk University \\ ${ }^{1}$ Department of Rehabilitation Science, Graduate School, Daegu University
}

Received: August 30, 2021 / Revised: August 30, 2021 / Accepted: September 26, 2021

(C) 2021 J Korean Soc Phys Med

\section{| Abstract |}

PURPOSE: This study examined the effects of action observational training with acoustic stimulation (AOTA) on the balance and gait ability in stroke patients.

METHODS: Forty-five chronic stroke patients were divided into three groups. The AOTA group $(n=15)$ received training via a video that showed a normal gait with the sound of footsteps. The action observation training (AOT) group ( $\mathrm{n}$ $=15$ ) received AOT without acoustic stimulation. The control group $(n=15)$ received physical training. Each intervention was applied once per day, three times per week for six weeks. The participants in the AOTA and AOT groups had five minutes of AOT. The participants in the all group had 20 minutes of physical training. All participants were measured using the Berg Balance Scale, the Timed Up and Go Test, the Functional Reaching Test, 10 Meter Walk Test, six Minute Walk Test, and Dynamic Gait Index. The collected data were analyzed using SPSS version 20.0 for Windows. The between- and within-group comparisons were analyzed using the one-way analysis of variance (ANOVA) test and

$\dagger$ Corresponding Author: Young Mi Kim

h1015m@naver.com, https://orcid.org/0000-0002-9343-6246

This is an Open Access article distributed under the terms of the Creative Commons Attribution Non-Commercial License (http://creativecommons.org/licenses/by-nc/3.0) which permits unrestricted non-commercial use, distribution, and reproduction in any medium, provided the original work is properly cited. a paired t-test, respectively. For all statistical analyses, the significance level was set to .05 .

RESULTS: The one-way ANOVA test identified significant differences among the measurement results of the three groups $(\mathrm{p}<.05)$. Post hoc analyses indicated the AOTA group to undergo more significant balance and gait changes than the control group $(\mathrm{p}<.05)$.

CONCLUSION: The gait and balance abilities could be improved effectively for patients with stroke when action observation training and acoustic stimulation were applied simultaneously.

Key Words: Action observation training, Acoustic stimulation, Balance, Gait, Stroke

\section{Introduction}

Stroke is a common cerebrovascular disease with a high mortality rate and persistent disability in adults worldwide. Stroke can cause motor insufficiency in terms of muscle weakness, spasticity, poor muscle coordination, and abnormal proprioception, which ultimately leads to walking disabilities [1-3]. In particular, stroke patients need to maintain balance while walking to perform independent walking [4]. The gait and dynamic balance abilities of stroke patients are crucial because of their close correlation with daily activities [5]. 
Various innovations have been applied to clinical practice to improve the walking and balancing skills of stroke patients. In particular, new therapeutic methods have been used in stroke rehabilitation, including task-oriented training methods focused on balance and movement [6], robot-assisted therapy [7], and virtual reality-based rehabilitation [8]. Existing rehabilitation programs for stroke patients focused mainly on direct physical training that restricts the non-paraplegic side and requires repetitive and active functional movements.

Recently, action observation training (AOT) using mirror neurons has been proposed as a useful intervention for recovering the functions of stroke patients with motor deficits [9]. AOT is a cognitive intervention to instill and improve various motor skills in patients with motor impairments, and it uses the characteristics of the mirror neurons that are excited when patients see others perform tasks or actual motor executions [10]. Moreover, AOT has been applied widely in limited spaces, making it a versatile intervention [9].

Several studies suggested that the mirror neurons play an important role in eliciting cortical motor activation and facilitating brain recovery in stroke patients [11-13], and provided evidence that AOT leads to a reorganization of the brain, which is associated with the motor learning cortex as the actual motor performance $[14,15]$.

Rhythmic acoustic stimulation (RAS) is the constant acoustic stimulation of rhythm that uses sound to influence the motor system and synchronize the motion and perceptual regions within a stable time, thus activating each brain region [16]. Rhythmical acoustic cuing, such as that achieved with beat music or metronomes, is used widely for gait rehabilitation in stroke patients [17,18]. Previous studies have shown that RAS is an effective intervention for improving balance and functional recovery in stroke patients. In particular, gait training and RAS are highly effective interventions for stroke patients.

Critically, while some studies have applied AOT with only visual stimulation, few studies have been conducted on AOT with audiovisual stimulation (AOTA), particularly AOT with RAS. Therefore, this study examined the effects of AOTA on the walking and balancing abilities of stroke patients and the differences between applying two sensory stimuli together and focusing only on a visual stimulus.

\section{Methods}

\section{Participants}

Forty-five patients hospitalized in S Nursing Hospital, P City, participated in this study. All subjects were fully informed of this study purpose and methodology, and they gave their consent before participation. The Bioethics Committee of Andong Science College approved this study (7003565-202001-HR-001-01). The selected participants were assigned randomly to the AOTA group $(\mathrm{n}=15)$, AOT group $(n=15)$, or control group $(n=15)$. The selection criteria were patients who had been diagnosed with a stroke and experienced its onset at least six months earlier, had no audiovisual impairments, scored more than 24 points on the Korean Mini-Mental State Examination (MMSE-K), and could walk more than $10 \mathrm{~m}$ independently or with assisted devices. The exclusion criteria were a positive history of neoplasms, cardiovascular disease, respiratory disease, clinically significant muscular-skeletal disease, other neurological conditions, uncorrected visual or auditory disturbances, or hospitalization in the previous three months.

\section{Measurement}

\section{1) Berg Balance Scale}

The Berg Balance Scale (BBS) is a 14-item objective measure that assesses a participant's ability to maintain balance while sitting, standing, and changing posture. The scale ranged from 0 , the minimum score for those who cannot perform the task given in an item, to 4 , the maximum 
score for those who can perform the task independently and completely. The maximum total score was 56 points, and scores from 0 to 20 represent balance impairment. The test had high reliability $(r=.98)$ [19].

\section{2) Timed Up and Go Test}

The Timed Up and Go Test (TUG) is a screening tool used to measure a participant's functional mobility and ability to transfer. During the TUG, each participant was seated on a chair, and on the word "go," they walked to the $3 \mathrm{~m}$ away mark and returned. Timing began on the word "go" and ended when the participant had sat down again. This test has high internal validity $(\mathrm{ICC}=.98)$ [20].

\section{3) Functional Reaching Test}

During the Functional Reach Test (FRT), each participant was asked to raise their arms and forearms to shoulder height. After achieving the metacarpal phalangeal (MCP) joint position, each participant was asked to perform their maximal forward and lateral reach while keeping their posture balanced. The difference between the initial and final positions of the MCP joint was calculated as their individual reaching capacity, and the score was recorded in centimeters. The FRT is an accepted clinical balance test in the elderly population and has demonstrated high testretest reliability in various adult populations $(r=.89 .92)$ [21].

\section{4) 10 Meter Walk Test}

The 10 Meter Walk Test (10MWT) was used to evaluate the gait ability. Each participant walked $14 \mathrm{~m}$ at a comfortable pace, and the first $2 \mathrm{~m}$ and last $2 \mathrm{~m}$ were excluded from the measurement due to acceleration and deceleration. The intra-measurer reliability of the test has an acceptable range (ICC $=.891 .00)$, and the inter-measurer reliability was fairly high $(\mathrm{ICC}=.87)$ [22].

\section{5) Six-Minute Walk Test}

The Six-Minute Walk Test (6 MWT) was used to identify walking endurance. The 6MWT evaluates the length and speed of walking for six minutes and assesses the walking ability required for daily activities. The test has high reliability $(r=.94)$ [23].

\section{6) Dynamic Gait Index}

The Dynamic Gait Index (DGI) was designed to evaluate the dynamic balance during walking. It has eight items that require the participants to maintain balance during normal walking and walking in different situations (e.g., changing speed, head turn, over and around obstacles, pivot turn, and stairs climbing). Each item was scored from 0 to 3 points, with a maximum total score of 24 . A high total DGI score indicates a high level of independent functional mobility. The test-retest reliability of the Korean version of the DGI was high $(r=.92)$, and the inter-measurer reliability was acceptable $(r=.88)$ [24].

\section{Procedure}

All training was conducted once per day, three times per week, for six weeks. The participants in the AOTA group had five minutes of AOT along with RAS and 20 minutes of physical training, whereas the participants in the AOT group had five minutes of AOT and 20 minutes of physical training. The AOT and AOTA groups showed equal contents of action observation for five minutes. The participants executed the physical training in the same video image environment for 20 minutes. The participants in the control group had only 20 minutes of physical training. All three groups received the same general physiotherapy treatment, which used the same action shown in the video. Acoustic stimulation was provided using a metronome at an average cadence after measuring the cadence per minute at a comfortable pace demonstrated by the subject in the video. The cues consisted of a rhythmical digital sound ("beep") emitted by a digital metronome, with a frequency ranging from 60 to $120 \mathrm{~Hz}$. The beep cadence was personalized and optimized for each patient during the first 


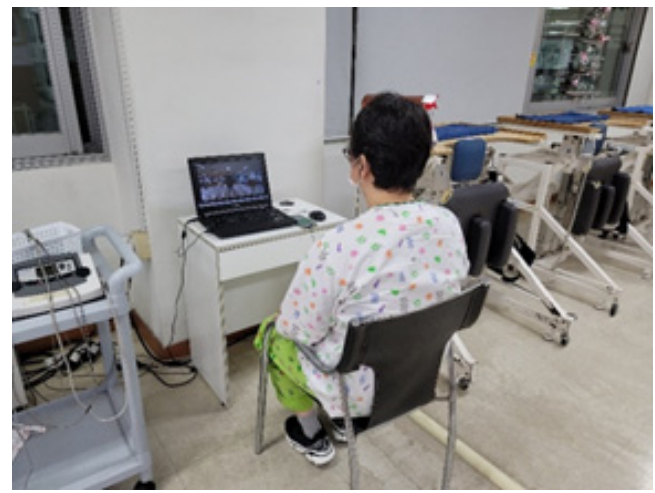

Fig. 1. Participant watching the functional walking tasks video with acoustic stimulation.

rehabilitative session by a physical therapist.

1) Rhythmic Acoustic Stimulation

Acoustic stimulation was applied according to the cadence of each subject using the Metronome app (Real/metronome, Gismart, UK) on a smartphone. Acoustic stimulation was provided via an individual earphone. Each participant observed the video and performed repeated training while listening to the acoustic stimulation. In particular, acoustic stimulation was individually customized before the experiment by studying the walking cadence per minute of each participant. To prevent confusion during training, different earphones were provided for each participant. Physical training was conducted while a skilled therapist observed from the participant's side to prevent possible accidents that could occur due to earphones (Fig. 1).

\section{2) Action Observation Physical Training}

The observation video for the AOTA and AOT groups consisted of a person lifting their heel from a standing position at a comfortable pace, moving from sitting to standing, tandem walking, figure-eight walking, and moving up and down stairs. The front and side of each motion were shot for 30 seconds each. Hence, each motion lasted for one minute, resulting in five minutes for five motions. After the observation, each participant used the

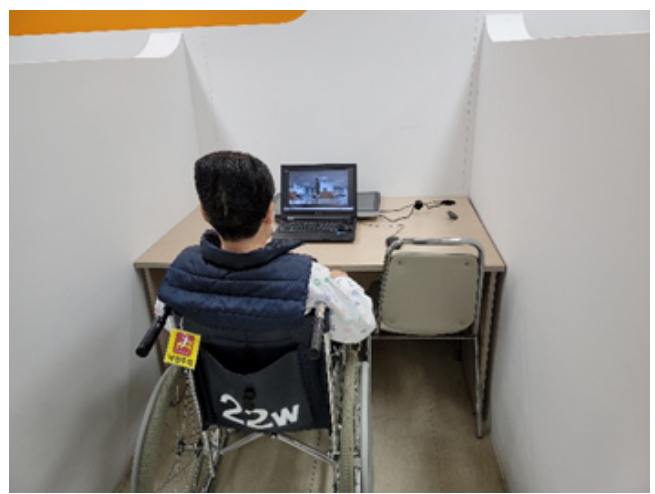

Fig. 2. Participant undergoing action observation training.

same motions for 20 minutes during their physical training (Fig. 2).

\section{3) Physical Training}

The physical training consisted of the same motions in the video: lifting a heel from a standing position at a comfortable pace, moving from sitting to standing, tandem walking, figure-eight walking, and moving up and down stairs (Fig. 3).

\section{Statistical Analysis}

The data were analyzed using SPSS ver. 20.0 (SPSS Inc., Chicago, II, USA). The homogeneity of the general characteristics of the participants is expressed using the mean and standard deviation, and the Kolmogorov-Smirnov test was applied to obtain a normal distribution. The between- and within-group comparisons were analyzed using the one-way analysis of variance (ANOVA) test and paired t-test, respectively. A Bonferroni correction was used as a post-test. $\mathrm{P}$ values $<.05$ were considered significant.

\section{Results}

\section{General Characteristics of the Participants}

Table 1 lists the participants' characteristics. No significant intergroup differences were found with respect 


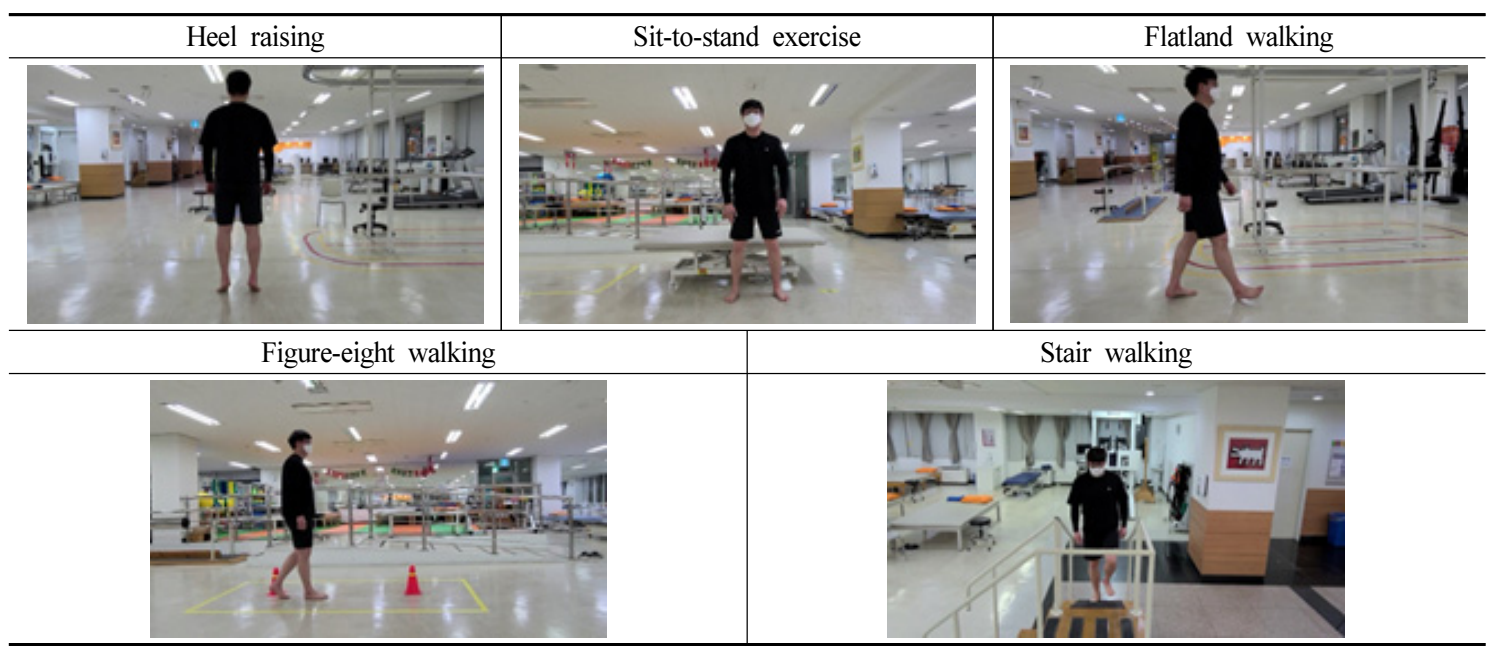

Fig. 3. Demonstration of the physical training exercises.

Table 1. General Characteristics of the Participants

\begin{tabular}{ccccccc}
\hline & AOTA & AOT & C & & $\mathrm{f}$ \\
\cline { 2 - 4 } & $\mathrm{M} \pm \mathrm{SD}$ & $\mathrm{M} \pm \mathrm{SD}$ & $\mathrm{M} \pm \mathrm{SD}$ & & & \\
\hline Gender (male/female) & $11 / 04$ & $10 / 05$ & $12 / 03$ & & .49 \\
Age (year) & $59.60 \pm 10.65$ & $62.47 \pm 10.03$ & $64.87 \pm 14.77$ & .72 & .79 & .46 \\
Height (cm) & $168.2 \pm 8.31$ & $157.53 \pm 39.37$ & $164.73 \pm 6.97$ & $62.35 \pm 9.39$ & 1.32 & .28 \\
Weight (kg) & $68.8 \pm 12.69$ & $66.34 \pm 10.62$ & 62.35 & .32 \\
MMSE-K (score) & $26.27 \pm 1.98$ & $25.93 \pm 1.94$ & $25.13 \pm 2.29$ & 1.17 & .32 \\
\hline
\end{tabular}

AOTA: action observation training with acoustic stimulation group

AOT: action observation training group

$\mathrm{C}$ : control group

to age, height, weight, or MMSE-K score $(p>.05)$.

IV. Discussion

\section{Comparison of the Clinical Test Results}

Table 2 lists the pre- and post-test balance and gait measurements for each group. One-way ANOVA revealed significant differences in the BBS, TUG, FRT, 10MWT, $6 \mathrm{MWT}$, and DGI scores among the three groups $(\mathrm{p}<.05)$. Post hoc analyses revealed the AOTA group to show more significant changes in the BBS, TUG, FRT, 10MWT, $6 \mathrm{MWT}$, and DGI scores than the control group $(\mathrm{p}<.05)$.
This study examined whether AOTA could improve the balance and gait ability in stroke patients compared to AOT and physical training. The results suggest that the therapeutic AOTA intervention used in this study may help improve balance and gait in stroke patients.

These results support those of previous studies on the gait function. Some studies concluded that walking training combined with acoustic cueing improved gait function significantly in stroke patients in terms of their TUG, BBS, and FMA scores and symmetrical step time [17,25]. In a similar 
Table 2. Comparison of the Clinical Test Results of the AOTA, AOT, and Control Groups

\begin{tabular}{|c|c|c|c|c|c|c|}
\hline & & AOTA $(\mathrm{n}=15)$ & AOT $(\mathrm{n}=15)$ & $C(\mathrm{n}=15)$ & $\mathrm{f}$ & $\mathrm{p}$ \\
\hline \multirow[t]{4}{*}{ BBS } & pre & $46.85 \pm 4.04$ & $44.79 \pm 6.07$ & $45.62 \pm 5.82$ & .494 & .614 \\
\hline & post & $50.23 \pm 3.39$ & $47.14 \pm 6.68$ & $47.23 \pm 5.54$ & & \\
\hline & Difference & $3.38 \pm 1.61^{\dagger}$ & $2.36 \pm 2.06$ & $1.62 \pm 1.50$ & 3.35 & $.046^{*}$ \\
\hline & $\mathrm{p}$ & $.000^{*}$ & $.001^{*}$ & $.001^{*}$ & & \\
\hline \multirow[t]{4}{*}{ TUG } & pre & $24.88 \pm 6.52$ & $24.92 \pm 10.79$ & $23.61 \pm 5.54$ & .112 & .885 \\
\hline & post & $22.01 \pm 5.51$ & $22.42 \pm 9.08$ & $22.82 \pm 5.61$ & & \\
\hline & Difference & $-2.87 \pm 2.81^{\dagger}$ & $-2.49 \pm 2.59$ & $-.79 \pm 1.08$ & 3.253 & $.049^{*}$ \\
\hline & $\mathrm{p}$ & $.001^{*}$ & $.003^{*}$ & $.017^{*}$ & & \\
\hline \multirow[t]{4}{*}{ FRT } & pre & $18.32 \pm 7.38$ & $18.96 \pm 6.25$ & $17.29 \pm 8.59$ & .19 & .827 \\
\hline & post & $22.49 \pm 6.89$ & $22.31 \pm 5.47$ & $19.35 \pm 8.69$ & & \\
\hline & Difference & $4.17 \pm 2.89^{\dagger}$ & $3.36 \pm 2.08$ & $2.13 \pm 1.17$ & 3.354 & $.045^{*}$ \\
\hline & $\mathrm{p}$ & $.000^{*}$ & $.000^{*}$ & $.000^{*}$ & & \\
\hline \multirow[t]{4}{*}{$10 \mathrm{~m}$} & pre & $19.55 \pm 8.21$ & $19.92 \pm 10.29$ & $18.95 \pm 9.22$ & .039 & .962 \\
\hline & post & $17.16 \pm 7.75$ & $18.36 \pm 9.86$ & $18.24 \pm 8.91$ & & \\
\hline & Difference & $-2.39 \pm 1.57^{\dagger}$ & $-1.57 \pm 1.12$ & $-.71 \pm 1.13$ & 5.915 & $.006^{*}$ \\
\hline & $\mathrm{p}$ & $.000^{*}$ & $.000^{*}$ & $.037^{*}$ & & \\
\hline \multirow[t]{4}{*}{$6 \min$} & pre & $182.83 \pm 77.7$ & $171.25 \pm 83.52$ & $200.35 \pm 89.06$ & .432 & .652 \\
\hline & post & $220.13 \pm 109.22$ & $192.64 \pm 81.71$ & $210.64 \pm 88.90$ & & \\
\hline & Difference & $37.33 \pm 45.78^{\dagger}$ & $21.39 \pm 13.73$ & $10.29 \pm 7.03$ & 3.315 & $.047^{*}$ \\
\hline & $\mathrm{p}$ & $.007^{*}$ & $.000^{*}$ & $.000^{*}$ & & \\
\hline \multirow[t]{4}{*}{ DGI } & pre & $9.40 \pm 4.03$ & $9.87 \pm 4.41$ & $12.00 \pm 4.04$ & 1.664 & .202 \\
\hline & post & $11.27 \pm 4.11$ & $10.8 \pm 3.89$ & $12.47 \pm 4.07$ & & \\
\hline & Difference & $1.87 \pm .92^{\dagger}$ & $.93 \pm .96$ & $.47 \pm .83$ & 9.306 & $.000^{*}$ \\
\hline & $\mathrm{p}$ & $.000^{*}$ & $.002^{*}$ & $.048^{*}$ & & \\
\hline
\end{tabular}

$\mathrm{M} \pm \mathrm{SD}:$ Mean \pm Standard Deviation

${ }^{*} \mathrm{p}<.05$, tsignificant difference in the change values between control groups

AOTA: action observation training with acoustic stimulation group

AOT: action observation training group

$\mathrm{C}$ : control group

vein, Song et al. showed that acoustic stimulation with visual stimuli improved the balance and gait function after eight weeks of training in patients with Parkinsonism [26].

Moreover, these results bolster the literature on RAS. In their study on RAS and treadmill gait training, Roerdink et al. reported that the gait parameters of their participants changed when the rhythmic acoustic frequency changed. In particular, the stride and step lengths were shortened, and the stride and step times decreased significantly [27]. Hyun and Choi examined 21 stroke patients for three weeks, and when comparing the backward walking with the RAS group and the backward walking training group, they reported that the RAS group had more significant differences in the TUG items used to assess the dynamic balance ability [28].

In this study, the AOTA group showed significant 
improvement in the balance and gait abilities, which is similar to the results of previous studies with the AOTA groups. The AOT group showed no significant differences compared to the control group. Ultimately, these results represent the effects of the balance reaction that the participants learned during the gait training procedure [29], and their balance improved because the training tasks, including the turning tasks, consisted of both the straight gait and oval-shaped gait.

In a four-week study on 30 stroke patients, Cho reported that the group that performed AOT along with rhythmic auditory training showed a significant difference in the functional gait assessment and 6MWT scores compared to the AOT group [30]. In contrast, the AOTA and AOT groups in this study did not show any significant differences in any of the items. In a study of stroke patients, Kim et al. reported that the group that conducted action observation and acoustic feedback training (AOTWA) improved its gait function more than the group that only conducted action observation and the control group. This suggests that the application of AOTWA is a useful therapeutic intervention. Furthermore, their study showed that the AOTWA group had significant differences in its 10MWT and DGI scores compared to those of the AOT and control groups. Moreover, both the AOTWA and AOT groups had significant differences in their 10MWT, DGI, TUG, and F8WT scores compared to those of the control group, which differs from the present study results [31].

One study reported that acoustic feedback training could be influential because it is related to all phases of cognition and facilitates motor learning [32]. Other studies proposed that regular acoustic stimulation evokes the entertainment stimulus to physical movements through the reticulospinal tract and visually structures a motor pattern in the brain, which allows for the training of functional muscle movements [33,34]. In addition, previous studies also showed that regular acoustic stimulation of patients with neurological disorders, such as stroke and Parkinson's disease, could improve their gait functions [35,36].

In the present study, the AOTA group demonstrated more changes after the intervention than the AOT group. Moreover, only the AOTA group showed significant differences compared to the control group. This suggests that providing visual observations with acoustic feedback can improve the functions of stroke patients more than visual observation alone.

The present study determined the effects of AOT and RAS on the gait and balance of stroke patients using an intervention that combined different stimuli. Critically, the functional gait and balance abilities were improved significantly in the experimental group that used audiovisual means. Consequently, the simultaneous application of sensory elements could have a strong positive impact on promoting motor learning and sensory movements, further facilitating plasticity in the central nervous system. Thus, the combined intervention may be an effective therapeutic method.

This study has several limitations. First, the results cannot be generalized to all stroke patients because the number of participants was small. Second, long-term follow-up studies were not conducted after the intervention. Thus, it is unclear how long the AOTA training will be effective. Accordingly, more diverse variables are needed to generalize the results. Further studies are required to overcome these limitations.

\section{Conclusion}

These findings suggest that AOTA may be an effective therapeutic approach to improve the balance and gait function in stroke patients. This was attributed to a cortical excitability change in the motor-related cortical areas.

\section{Acknowledgments}

This work was supported by the National Research 
Foundation of Korea (NRF) and a grant funded by the Korean government (2020R1I1A1A01053491).

\section{References}

[1] Garland SJ, Gray VL, Knorr S. Muscle activation patterns and postural control following stroke. Motor Control. 2009;13(4):387-411.

[2] Langhorne P, Coupar F, Pollock A. Motor recovery after stroke: a systematic review. Lancet Neurol. 2009;8(8): 741-54.

[3] Li S, Liu J, Bhadane M, et al. Activation deficit correlates with weakness in chronic stroke: evidence from evoked and voluntary EMG recordings. Clin Neurophysiol. 2014; 125(12):2413-7.

[4] Ham SC, Lim GJ. The effects of robot-assisted gait training with visual feedback on gait, balance and balance confidence in chronic stroke patients. J Kor Phy Ther. 2016;28(2):71-6.

[5] Langhammer B, Stanghelle JK. Co-variation of tests commonly used in stroke rehabilitation. Physiother Res Int. 2006;11(4):228-34.

[6] Kuberan P, Kumar V, Joshua AM, et al. Effects of task oriented exercises with altered sensory input on balance and functional mobility in chronic stroke: a pilot randomized controlled trial. Int J Rehabil Res. 2017; 16(2):307-13.

[7] Chisari C, Bertolucci F, Monaco V, et al. Robot-assisted gait training improves motor performances and modifies Motor Unit firing in poststroke patients. Eur J Phys Rehabil Med. 2015;51(1):59-69.

[8] Cho KH, Lee KJ, Song CH. Virtual-reality balance training with a video-game system improves dynamic balance in chronic stroke patients. Tohoku J Exp Med. 2012; 228(1):69-74.

[9] Zhang JJ, Fong KN, Welage N, et al. The activation of the mirror neuron system during action observation and action execution with mirror visual feedback in stroke: a systematic review. Neural Plast. 2018.

[10] Rizzolatti G, Fogassi L, Gallese V. Neurophysiological mechanisms underlying the understanding and imitation of action. Nat Rev Neurosci. 2001;2(9):661-70.

[11] Franceschini M, Ceravolo MG, Agosti M, et al. Clinical relevance of action observation in upper-limb stroke rehabilitation: a possible role in recovery of functional dexterity. A randomized clinical trial. Neurorehabil Neural Repair. 2012;26(5):456-62.

[12] Garrison KA, Aziz-Zadeh L, Wong SW, et al. Modulating the motor system by action observation after stroke. Stroke. 2013;44(8):2247-53.

[13] Harmsen WJ, Bussmann JB, Selles RW, et al. A mirror therapybased action observation protocol to improve motor learning after stroke. Neurorehabil Neural Repair. 2015;29(6):509-16.

[14] Gatti R, Tettamanti A, Gough PM, et al. Action observation versus motor imagery in learning a complex motor task: a short review of literature and a kinematics study. Neurosci Lett. 2013;540:37-42.

[15] Patel M. Action observation in the modification of postural sway and gait: Theory and use in rehabilitation. Gait Posture. 2017;58:115-20.

[16] Thaut MH, Stephan KM, Wunderlich G, et al. Distinct cortico-cerebellar activations in rhythmic auditory motor synchronization. Cortex. 2009;45(1):44-53.

[17] Park J, Park Sy, Kim Yw, et al. Comparison between treadmill training with rhythmic auditory stimulation and ground walking with rhythmic auditory stimulation on gait ability in chronic stroke patients: A pilot study. NeuroRehabilitation. 2015;37(2):193-202.

[18] Shin YK, Chong HJ, Kim SJ, et al. Effect of rhythmic auditory stimulation on hemiplegic gait patterns. Yonsei Med J. 2015;56(6):1703-13.

[19] Blum L, Korner-Bitensky N. Usefulness of the Berg Balance Scale in stroke rehabilitation: a systematic review. Phys Ther. 2008;88(5):559-66.

[20] Morris S, Morris ME, Iansek R. Reliability of measurements 
obtained with the Timed "Up \& Go" test in people with Parkinson disease. Phys Ther. 2001;81(2):810-8.

[21] Lin YH, Tang YW, Chen TR, et al. A reliability study for standing functional reach test using modified and traditional rulers. Percept Mot Skills. 2012;115(2):512-20.

[22] Dobkin BH. Rehabilitation after stroke. New England Journal of Medicine. 2005;352(16):1677-84.

[23] Mossberg KA. Reliability of a timed walk test in persons with acquired brain injury. Am J Phys Med Rehabil. 2003;82(5):385-90.

[24] An S, Seo H, Chung YJ. Reliability and validity the Korean version of the dynamic gait index in patients with stroke. Journal of Special Education \& Rehabilitation Science. 2011;50(2):289-306.

[25] Shin YI, Yang SH, Kim JY. Clinical feasibility of wearable robot orthosis on gait and balance ability for stroke rehabilitation: a case study. J Kor Phys Ther. 2015; 27(2): 124-7.

[26] Song J, Zhou P, Cao Z, et al. Rhythmic auditory stimulation with visual stimuli on motor and balance function of patients with Parkinson's disease. Eur Rev Med Pharmacol Sci. 2015;19(11):2001-7.

[27] Roerdink M, Lamoth CJ, Kwakkel G, et al. Gait coordination after stroke: benefits of acoustically paced treadmill walking. Phys Ther. 2007;87(8):1009-22.

[28] Hyun DS, Choi JD. The effects of backward walking with rhythmic auditory stimulation on gait and balance in patients with stroke. Journal of the Korea AcademiaIndustrial cooperation Society. 2013;14(12): 6237-45.
[29] Baram Y, Miller A. Auditory feedback control for improvement of gait in patients with Multiple Sclerosis. J Neurol Sci. 2007;254(1-2):90-4.

[30] Cho HY. Effect of balance and gait in stroke patient with action observation using audiovisual. Master's Degree. Daegu University. 2017.

[31] Kim HM, Son SM. Effect of action observation training with auditory feedback for gait function of stroke patients with hemiparesis. J Kor Phys Ther. 2017;29(5):246-54.

[32] Thielman G, Kaminski T, Gentile AJ, et al. Rehabilitation of reaching after stroke: comparing 2 training protocols utilizing trunk restraint. Neurorehabil Neural Repair. 2008;22(6):697-705.

[33] Whitall J, Waller SM, Silver KH, et al. Repetitive bilateral arm training with rhythmic auditory cueing improves motor function in chronic hemiparetic stroke. Stroke. 2000;31(10):2390-5.

[34] Thaut M, Kenyon G, Hurt C, et al. Kinematic optimization of spatiotemporal patterns in paretic arm training with stroke patients. Neuropsychologia. 2002;40(7):1073-81.

[35] Rochester L, Hetherington V, Jones D, et al. The effect of external rhythmic cues (auditory and visual) on walking during a functional task in homes of people with Parkinson's disease. Arch Phys Med Rehabil. 2005; 86(5):999-1006.

[36] Hayden R, Clair AA, Johnson G, et al. The effect of rhythmic auditory stimulation (RAS) on physical therapy outcomes for patients in gait training following stroke: a feasibility study. Int J Neurosci. 2009;119(12):2183-95. 